DOI: $10.26461 / 17.04$

\title{
Diseño, construcción y evaluación de un analizador autónomo in situ para la monitorización de los niveles de fósforo y nitrógeno en aguas superficiales. Primeros resultados
}

Design, construction and evaluation of an in situ autonomous analyser for monitoring of phosphorus and nitrogen levels in surface waters. First results

RECIBIDO: 15/10/2018 $\rightarrow$ APROBADO: 7/11/2018 \mknochen@fq.edu.uy

i̊ KNochen, moisés $(1,3)$; Roth, gulllermo $(1,3)$; GOnZÁlez, pablo $(1,3)$; PÉREZ, NiCOLÁS $(2,3)$; MONZÓN, PABlo (2); DEl CASTILlo, MARIANA (2)

(1) Departamento estrella campos, facultad de química, universidad DE LA REPÚBLICA, MONTEVIDEO, URUGUAY.

(2) INSTItUto DE INGENIERÍA ELÉCTRICA, FACUlTAd DE INGENIERÍA, UNIVERSIDAD DE LA REPÚblica, MONTEVIDEO, URUguay.

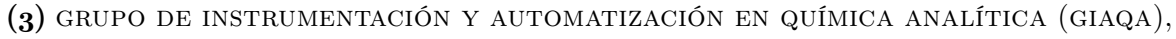
FACULTAD DE QUímiCA, UNIVERSIDAD DE LA REPÚBLICA, MONTEVIDEO, URUGUAY.

\section{RESUMEN}

Se describe un prototipo de analizador automatizado de bajo costo capaz de operar in situ junto a un cuerpo de agua realizando la determinación analítica de los niveles de fósforo (ortofosfato) y nitrógeno (nitrato + nitrito), así como $\mathrm{pH}$ y temperatura, transmitiendo los resultados obtenidos a una estación remota mediante internet inalámbrica. Las determinaciones de fósforo y nitrógeno son realizadas mediante métodos de desarrollo de color con detección fotométrica implementados en un sistema de flujo pulsado. Para el control y adquisición de datos se recurrió a la plataforma de microcontroladores Arduino. El sistema opera con baterías recargables y habilita el uso de un panel solar para su recarga. La evaluación preliminar de los métodos analíticos realizada en el laboratorio arrojó cifras de mérito perfectamente adecuadas para los fines buscados. Está prevista una evaluación de campo donde se estudiará el desempeño del equipo en condiciones reales. Se entiende que el uso de este tipo de sistemas que no requieren personal para su operación puede resultar de utilidad en el análisis ambiental.

PAlAbRAS ClAVE: Agua, nutrientes, análisis automatizado, telemetría química. 


\section{ABSTRACT}

A prototype of a low-cost automated analyser capable of in situ operation next to a water body is described. The analyser can determine levels of phosphorus (orthophosphate) and nitrogen (nitrate + nitrite), along with $\mathrm{pH}$ and temperature, transmitting the results to a remote station via wireless internet. Phosphorus and nitrogen determinations are carried out by means of photometric methods, implemented in a pulsed-flow system. For control and data acquisition the open-source Arduino platform of microcontrollers was used. The system runs on rechargeable batteries, with the possible support of a solar panel. Preliminary evaluation of the analytical methods carried out in the laboratory showed figures of merit fit for the purpose. A field evaluation is foreseen in the near future, where the analyser will be tested under real conditions. This type of instruments is considered to be potentially useful in environmental analysis.

KEYWORDS: Water, nutrients, automated analysis, chemical telemetry.

\section{INTRODUCCIÓN}

\section{Analizadores in situ y telemetría química}

La evaluación del estado de los cuerpos de agua desde el punto de vista de su calidad es un área en la que el análisis químico y físicoquímico encuentra un amplio campo de aplicación. Esto es cierto en lo relativo a su aptitud para el uso (riego, recreación, fuente de agua para consumo humano), a los aspectos regulatorios y también en estudios científicos en el campo de las ciencias ambientales.

Las tecnologías analíticas disponibles en la actualidad permiten la determinación en las muestras ambientales de un gran número de sustancias a niveles de concentración cada vez más bajos y con gran precisión y exactitud. Como consecuencia la demanda de análisis y resultados relativos a la calidad ambiental ha aumentado considerablemente, con la consiguiente sobrecarga de los laboratorios. Se debe tener presente que la estrategia tradicional empleada en el análisis de muestras ambientales (al igual que la mayoría de las matrices) está centrada en el laboratorio: se extraen muestras en los puntos designados, que son transportadas al laboratorio y almacenadas hasta el instante del análisis. A diferencia de otros campos de aplicación del análisis químico, como el control de calidad industrial, en el análisis ambiental los puntos de muestreo suelen estar a gran distancia del laboratorio y en lugares no siempre de fácil acceso. Por lo tanto, la estrategia tradicional exige no solo una planificación ajustada sino un despliegue logístico para transportar el personal encargado de la toma de muestra y su equipo hasta el punto de muestreo y de regreso. Las muestras deben ser aditivadas y refrigeradas para garantizar su conservación y luego transportadas al laboratorio, donde serán almacenadas hasta la realización del análisis. Este modo de operación presenta entonces costos adicionales asociados al muestreo. Además, sobre todo en el caso del análisis a niveles de trazas, existe 
el riesgo de alteración de la muestra durante el transporte y almacenamiento, ya sea por contaminación o por pérdidas de analito.

La estrategia alternativa basada en el empleo in situ de kits de reactivos logra evitar el transporte, la conservación y el almacenamiento de muestras, pero sigue requiriendo del desplazamiento del personal para la realización de los análisis de campo.

Los avances tecnológicos surgidos en las últimas décadas han dado lugar a una tercera estrategia, que implica un verdadero cambio de paradigma: el empleo de analizadores automatizados desplegados en campo en los lugares donde se desea obtener los datos analíticos. Al añadir al analizador la capacidad de establecer comunicaciones inalámbricas se hace posible enviar los resultados analíticos a una estación central. Se alcanza así la capacidad de realizar la telemedición o telemetría de los parámetros de interés y de obtener incluso resultados en tiempo real (Mukhopadhyay y Mason, 2013). La telemetría química es una tecnología ampliamente conocida en la industria de procesos químicos, e incluso en los últimos años también en la exploración espacial, posibilitando el análisis de la composición química de suelo y rocas en planetas como Marte (Grotzinger, et al., 2012). Últimamente esta disciplina está tomando auge también en el análisis ambiental (Glasgow, et al., 2004). La creciente disponibilidad comercial de sensores químicos para distintas sustancias y parámetros ha hecho posible la implementación de analizadores in situ que pueden ser instalados en campo y operan en forma desatendida reportando los resultados mediante un sistema de comunicaciones adecuado, ya sea con una periodicidad predeterminada o a demanda.

La telemetría no debe confundirse con la teledetección o percepción remota, en la cual el sensor está ubicado a cierta distancia del punto bajo estudio y, por tanto, sin contacto físico con él, como en el caso del empleo de aeronaves o satélites artificiales en monitorización ambiental. En la telemetría in situ el dispositivo sensor o analizador se ubica físicamente en el punto bajo estudio, distante del observador o usuario de la información.

Como se mencionó anteriormente, en la actualidad existe un amplio conjunto de sensores para varios parámetros químicos y físicoquímicos (Gründler, 2007; Narayanaswamy y Wolfbeis, 2004). No obstante, si empleamos el término sensores, según la definición estricta propuesta por IUPAC (Hulanicki, et al., 1991), existen numerosos parámetros químicos para los cuales actualmente no se dispone de sensores adecuados. A modo de ejemplo, no existen en la actualidad sensores comerciales para fosfato suficientemente confiables para ser empleados fuera del laboratorio. En estos casos es necesario recurrir a dispositivos más complejos, denominados analizadores, que implementan en forma completa el proceso analítico deseado.

Actualmente existe oferta comercial de varios tipos de analizadores in situ para el análisis de cuerpos de agua. Estos adoptan varias formas, por ejemplo, boyas (destinadas a flotar ancladas en los cuerpos de agua), cilindros (diseñados para ser sumergidos), etcétera. Algunos de estos sistemas se alimentan mediante baterías recargadas por paneles solares para asegurar su autonomía. Lamentablemente, 
su precio es elevado, lo cual puede ser un obstáculo a la hora de emplearlos en proyectos de bajo presupuesto.

En este contexto y con la intención de generar conocimiento local en un área poco desarrollada en el medio, nuestro grupo encaró el diseño y construcción de un prototipo de analizador in situ que pudiera ser usado en proyectos de investigación donde se requiera el despliegue de cierto número de analizadores para la determinación de varios parámetros de calidad en aguas superficiales.

\section{Objetivos y características del prototipo}

El objetivo fue diseñar y construir un prototipo de analizador in situ de bajo costo de construcción, con capacidad multiparamétrica y de comunicaciones, aprovechando las redes GSM ampliamente difundidas en el país.

Se definió que los parámetros a determinar serían ortofosfato, nitrato + nitrito, pH y temperatura. Estos fueron seleccionados por su papel en el desarrollo explosivo de poblaciones de cianobacterias, conocidos como "floraciones algales". Sin embargo, se previó que el sistema tuviera características modulares de modo de posibilitar la incorporación de subsistemas para la determinación de otros analitos o parámetros físicoquímicos de interés.

Para simplificar el diseño se decidió que en una primera etapa el prototipo debería poder determinar ortofosfato o nitrato + nitrito, pero no los dos simultáneamente. Para una etapa posterior se previó la posibilidad de determinar ambos parámetros en forma sucesiva.

\section{MATERIALES Y MÉTODOS}

\section{Subsistema de toma de muestra y medida de pH y temperatura}

Para cada determinación la muestra es tomada del cuerpo de agua mediante un tubo de goma de silicona y una minibomba peristáltica de $12 \mathrm{~V}$, pasando por un prefiltro grueso de malla y un filtro de membrana de celulosa. La muestra filtrada pasa por la celda de flujo que contiene el sensor de temperatura y el electrodo de $\mathrm{pH}$ y de allí a un reservorio intermedio con desborde. Desde este reservorio la muestra es bombeada por el subsistema analítico para la determinación de ortofosfato o de nitrato + nitrito.

La medida de $\mathrm{pH}$ se realiza mediante un electrodo de vidrio combinado ubicado en la celda de flujo.

Para la medida de temperatura del agua se emplea un sensor digital DS18B20 (Maxim) encapsulado en una vaina de acero inoxidable y ubicado en la misma celda de flujo del electrodo de $\mathrm{pH}$. 
La Figura 1 muestra el esquema de este subsistema.

SSA

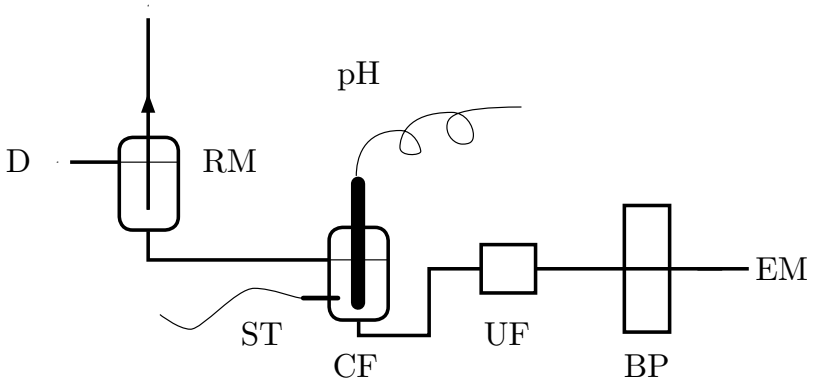

Figura 1. Esquema del subsistema de toma de muestra. EM: entrada de muestra. BP: bomba peristáltica. UF: unidad de filtración. $\mathrm{CF}$ : celda de flujo. $\mathrm{pH}$ : electrodo combinado de $\mathrm{pH}$. $\mathrm{ST}$ : sensor de temperatura. RM: reservorio de muestra. D: descarte. SSA: subsistema analítico.

\section{Subsistema analítico}

Para la determinación de ortofosfato así como de nitrato + nitrito se optó por métodos de desarrollo de color clásicos con detección fotométrica, implementados mediante sistemas en flujo (Estela y Cerdà, 2005; Miró, et al., 2003; Motomizu y Li, 2005). El ortofosfato se determina mediante el método del molibdovanadofosfato con medida a $365 \mathrm{~nm}$. Para nitrato+nitrito se recurrió al método de Griess con medida a 525 $\mathrm{nm}$. El nitrato es pre-reducido a nitrito en una minicolumna de cadmio cobreado.

El subsistema analítico para la determinación de ortofosfato y nitrato + nitrito se basa en el uso de flujo pulsado generado por microbombas solenoide con un desplazamiento de $20 \mu \mathrm{L}$ por pulso. Las mismas son alimentadas con pulsos de 12 $\mathrm{V}$ de ancho controlado enviados por el microcontrolador.

Para la detección fotométrica se emplearon LEDs como fuente de radiación y fotodiodos como detectores. Para el sistema de fosfato se empleó un LED ultravioleta de $365 \mathrm{~nm}$ (Nichia). El sistema de nitrato + nitrito emplea un LED de $525 \mathrm{~nm}$ (Kingbright). En ambos casos se emplearon celdas de flujo de cuarzo de $10 \mathrm{~mm}$ de camino óptico con un volumen interno de $80 \mu \mathrm{L}$. Estas se conectaron entre sí en serie, de modo que el flujo pasa primero por la celda de detección de nitrógeno y luego por la de fósforo. Las burbujas que puedan aparecer durante el proceso son eliminadas antes de ingresar a las celdas de flujo mediante un desgaseador basado en una membrana microporosa de Teflon. La Figura 2 muestra el diagrama general del subsistema analítico. 


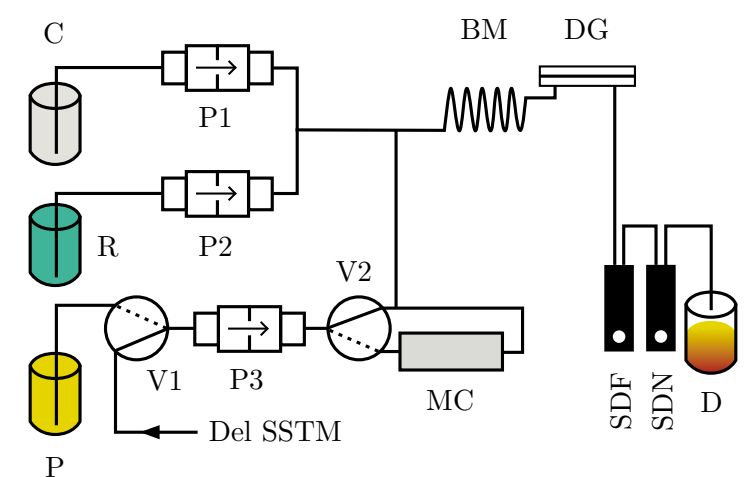

Figura 2. Esquema del subsistema analítico. P1, P2, P3: microbombas solenoide. V1, V2: válvulas solenoide. C: reservorio de portador. R: reservorio de reactivo. P: reservorio de patrón. MC: minicolumna rellena con gránulos de cadmio cuperizado. BM: bobina de mezclado. DG: desgaseador. SDF: sistema de detección para fósforo. SDN: sistema de detección para nitrógeno. D: descarte. SSTM: subsistema de toma de muestra.

\section{Electrónica}

Para el control del sistema se emplearon placas de microcontrolador de la plataforma Arduino (ver página web sugerida luego de las referencias; Colaboradores de Wikipedia, [s.d.]). El control global del sistema está a cargo de una placa Arduino Uno, que se comunica por medio de protocolo $\mathrm{I}^{2} \mathrm{C}$. Esta placa realiza también la medida de $\mathrm{pH}$ mediante una entrada analógica de 10 bits, y se comunica con el sensor de temperatura mediante protocolo 1-Wire. En el caso del electrodo de $\mathrm{pH}$ se implementó la conexión mediante un amplificador operacional de alta impedancia de entrada.

El subsistema analítico es controlado por una segunda placa Arduino Uno. Para la adquisición de datos se empleó una interfaz analógico-digital ADS1115 de 16 bits que se comunica con el Arduino mediante $\mathrm{I}^{2} \mathrm{C}$. La señal de los fotodiodos es amplificada mediante sendos amplificadores operacionales TL081 operados en el modo de transimpedancia. La activación de las microbombas solenoide se realiza desde salidas digitales del Arduino a través de un circuito integrado ULN2803, el cual también controla la activación de los LEDs asociados a la detección.

\section{Comunicaciones}

Las comunicaciones se realizan mediante un modem GSM/GPRS basado en el módulo SIM900, el cual se comunica con el microcontrolador central. Este modem ampliamente difundido permite implementar fácilmente comunicaciones mediante mensajes de texto (SMS) y también conexión a internet mediante GPRS. 


\section{Alimentación eléctrica}

La alimentación se implementó mediante baterías recargables. Dependiendo del periodo de autonomía deseado está previsto el reemplazo periódico de la batería por una ya cargada o bien recargar la batería mediante un panel solar.

\section{Construcción}

Para la construcción de partes de los subsistemas analítico y de toma de muestra se recurrió extensivamente a la impresión 3D mediante tecnología de filamento fundido (FFF). Se empleó un impresor 3D QidiTech X-one 2. Para las partes en contacto con agua o con reactivos se empleó filamento de ABS, mientras que para las partes accesorias se usó filamento de PLA.

El sistema se instala dentro de una caja de material plástico resistente, que alberga dos placas de acrílico ubicadas en dos niveles. El nivel superior se destinó a los subsistemas analítico, de toma de muestra y de medida de $\mathrm{pH}$ y temperatura. El nivel inferior se destinó a la electrónica (albergada en cajas estancas grado IP65), batería de alimentación y frascos de reactivos, patrones y descarte. La Figura 3 muestra una vista de la placa superior.

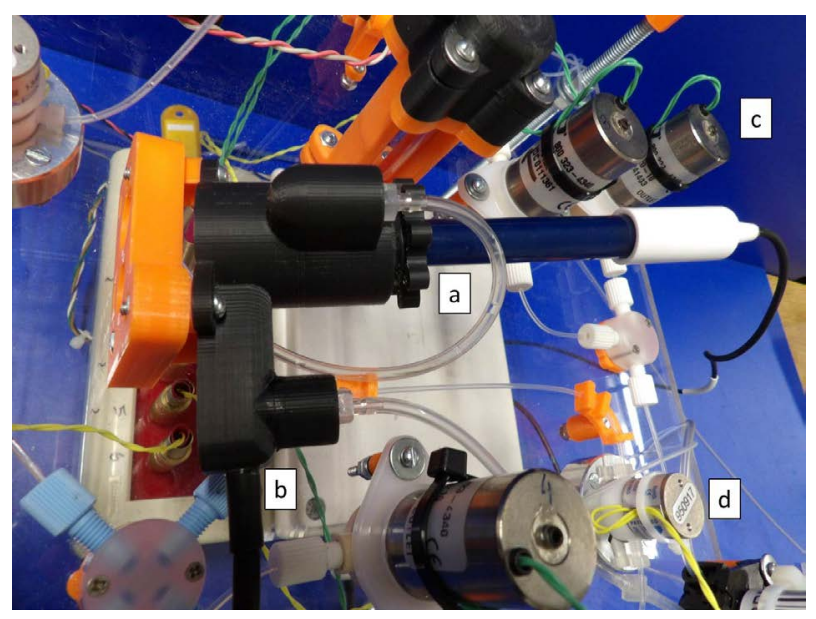

Figura 3. Vista de la placa de los subsistemas analítico y de toma de muestra. a: electrodo combinado de $\mathrm{pH}$ instalado en la celda de flujo. b: sensor de temperatura. c: microbombas solenoide. d: válvula solenoide.

\section{RESULTADOS}

\section{Validación de métodos}

En una primera etapa se validaron en el laboratorio los métodos para fosfato y nitrato + nitrito. Posteriormente se realizarán las pruebas de campo, donde se validarán 
todos los métodos y se tomarán en forma paralela y simultánea contramuestras que serán analizadas en el laboratorio por métodos de referencia.

La validación incluyó la precisión (repetibilidad), el rango lineal y los límites de detección y cuantificación. La precisión se evaluó calculando la desviación estándar relativa de la altura de pico obtenida en 10 inyecciones sucesivas.

\section{Validación en el laboratorio del método para $\mathrm{PO}_{4}^{3-}$}

La precisión $\left(\mathrm{s}_{\mathrm{r}}(\%)\right)$ para la determinación de fosfato a la concentración de $1 \mathrm{mg}-\mathrm{P}$ $\mathrm{L}^{-1}$ fue mejor que $1 \%$, y de $4,5 \%$ a la concentración de $0,1 \mathrm{mg}-\mathrm{P} \mathrm{L}^{-1}$.

Para la evaluación del rango lineal se inyectaron por triplicado soluciones en el rango de 0 a $20 \mathrm{mg}-\mathrm{P} \mathrm{L}^{-1}$. Se verificó la hipótesis de linealidad a concentraciones hasta $10 \mathrm{mg}-\mathrm{P} \mathrm{L}^{-1}\left(\mathrm{R}^{2}=0,997\right)$.

Los límites de detección (3s) y cuantificación (10s) fueron 5,5 y 18,2 $\mu \mathrm{g}-\mathrm{P} \mathrm{L}^{-1}$, equivalentes a 16,9 y $55,8 \mu \mathrm{g}-\mathrm{PO}_{4}^{-} \mathrm{L}^{-1}$, respectivamente.

\section{Validación en el laboratorio del método para nitrato+nitrito}

La precisión $\left(\mathrm{s}_{\mathrm{r}}(\%)\right)$ fue de $1,8 \%$ a $0,8 \mathrm{mg}-\mathrm{N} \mathrm{L}^{-1}$.

La linealidad fue evaluada inyectando por triplicado soluciones de patrón nitra-

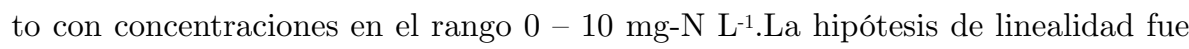
verificada en el rango hasta $4 \mathrm{mg}^{-\mathrm{N} \mathrm{L}^{-1}}\left(\mathrm{R}^{2}=0,994\right)$.

Los límites de detección (3s) y cuantificación (10s) fueron de 4,3 y 14,2 $\mu \mathrm{g}-\mathrm{N}$ $\mathrm{L}^{-1}$, respectivamente.

Asimismo, se estudió la vida útil de la columna de cadmio cuperizado empleada para la determinación de nitrato, y se halló que su eficiencia disminuyó de $90 \%$ a $74 \%$ luego de 340 ciclos de análisis.

\section{DISCUSIÓN}

\section{Metodologías analíticas}

Una de las primeras decisiones que se debían tomar fue la de los parámetros a determinar. Si bien para determinar el contenido de fósforo en agua se prefiere con frecuencia determinar fósforo total, se encontró que los métodos de oxidación química se realizan a alta temperatura por lo que se requiere una gran cantidad de energía, incompatible con los postulados de autonomía establecidos. Por otra parte, las especies de fósforo que sirven como nutrientes son las solubles, por lo que se optó por determinar ortofosfato.

En cuanto a las técnicas analíticas, se consideró la posibilidad de emplear sensores potenciométricos. Si bien existe amplia experiencia con los electrodos selectivos a nitrato, los electrodos para fosfato son aún experimentales y no existe oferta significativa a nivel comercial. Por este motivo se optó por un método químico con detección fotométrica para la determinación de fosfato. 
Sin embargo, se evaluó el comportamiento de un electrodo combinado selectivo a nitrato (Thermo Orion) instalado en una celda de flujo y se emplearon soluciones patrón de diversas concentraciones de nitrato. Los resultados obtenidos sugieren que, si bien el desempeño es adecuado a altas concentraciones, tanto la linealidad de la calibración como la repetibilidad fueron pobres a bajas concentraciones como las que se espera determinar. A su vez, la necesidad de calibración multipunto aumentaría la complejidad del sistema. Por este motivo se optó también para el caso del nitrato por el uso de un método químico con detección fotométrica.

Entre las ventajas de estos métodos se encuentran la amplia experiencia acumulada, el detallado conocimiento de las posibles interferencias y la posibilidad de calibración con un único patrón dentro del rango de linealidad. A cambio de esas ventajas se requiere disponer de uno o más reactivos y de un control riguroso de los tiempos de reacción.

En el caso de la determinación de fosfato, el método más ampliamente usado en los laboratorios es el del llamado "azul de fosfomolibdeno". Se basa en una reacción compleja que, dependiendo de las condiciones de trabajo, puede dar lugar a diversos productos de reacción (Nagul, 2015). Si bien es uno de los métodos fotométricos más sensibles, la fuerte dependencia con las condiciones experimentales puede resultar inconveniente. Además, requiere reactivos reductores intrínsecamente inestables, como el ácido ascórbico.

Otro método disponible es el del ácido molibdovanadofosfórico (Kitson y Mellon, 1944; Abbott, et al., 1963). Este método puede desarrollarse empleando un único reactivo muy estable. Aunque es considerado como un método poco sensible, esto es consecuencia de que los manuales de métodos suelen indicar que la medida se realice a longitudes de onda de $400 \mathrm{~nm}$ o incluso superiores. Dado que el máximo de absorción del producto coloreado se encuentra a alrededor de $315 \mathrm{~nm}$, es fácil comprender que midiendo en un flanco de la banda de absorción a $400 \mathrm{~nm}$, la sensibilidad será muy baja. En este caso se exploró la conveniencia de usar una longitud de onda más próxima a la del máximo. Actualmente existe un fuerte desarrollo de diodos emisores de luz (LEDs) para la región del ultravioleta, aunque para las longitudes de onda más bajas estos son aún costosos y de baja potencia radiante. Como solución de compromiso se empleó un LED de 365 nm, con lo cual se alcanzó una sensibilidad aceptable para el propósito.

La selección de una longitud de onda menor a $400 \mathrm{~nm}$ presenta como inconveniente un mayor riesgo de interferencias espectrales. Por este motivo se incorporó en el programa una etapa de medida del "blanco de muestra" para corregir esas interferencias. Analizando muestras reales en la etapa de evaluación del prototipo en campo se espera obtener suficientes datos para comprobar la eficacia de esta estrategia.

Cabe señalar que la sensibilidad y detectabilidad exhibidas por el método para nitrógeno lo hacen adecuado al uso, considerando el valor máximo (10 mg-N L-1) establecido para los cuerpos de agua de clases 1 a 3 por el Decreto 253/79 (Uruguay, 1979).

En el caso del método para fósforo, las cifras de mérito exhibidas no son suficientes para permitir la determinación a niveles inferiores al valor máximo $\left(25 \mu \mathrm{g}-\mathrm{P} \mathrm{L}^{-1}\right)$ establecido para los cuerpos de agua de clases 1 a 3 por el mismo decreto (Uruguay, 
1979). Sin embargo resulta adecuado para la determinación de fósforo en cuerpos de agua contaminados, por ejemplo en la proximidad de puntos de vertido. En el futuro se podrá estudiar la mejora de esos parámetros.

\section{Determinaciones de $\mathrm{pH}$ y temperatura}

Estas determinaciones fueron incorporadas para complementar la información obtenida sobre los niveles de nutrientes. La determinación de la temperatura del agua es empleada además para corregir la medida de $\mathrm{pH}$.

Esta última, a pesar de su aparente simplicidad, encierra varias dificultades que deberán ser evaluadas en las pruebas de campo. Entre ellas, se encuentra la frecuencia con que deberá repetirse el ajuste (o calibración) del sistema de medición. Para no aumentar la complejidad del sistema en la primera versión del prototipo no se ha previsto realizar ese ajuste en forma autónoma, sino por un operador y en forma periódica, simultáneamente con la reposición de reactivos y cambio de batería. De esta forma se evita la necesidad de disponer de patrones de pH intrínsecamente poco estables.

\section{Electrónica y comunicaciones}

La plataforma de microcontroladores Arduino fue seleccionada por estar ampliamente disponible y ser de muy bajo costo. Esto último es consecuencia de ser una plataforma de "fuente abierta", lo que elimina los costos de propiedad intelectual. Asimismo, existe una extensa comunidad de desarrolladores que contribuyen en forma accesible con su conocimiento bajo la forma de bibliotecas, subrutinas y, en general, su experiencia.

La mayoría de las placas de dicha plataforma incorporan un puerto de comunicaciones USB, de modo que la carga y modificación de sus programas se puede realizar en forma muy simple desde un notebook, incluso en campo.

El sistema basado en microcontroladores no solo realiza el control de las bombas y válvulas del subsistema analítico sino que también controla la adquisición de datos fotométricos. Esta última consta de varias etapas. Al inicio de una secuencia de medición se ajusta la escala fotométrica, para ello se apaga el LED en uso y se mide y memoriza la "señal oscura", $\mathrm{S}_{\mathrm{D}}$. A continuación se enciende el LED y se llena la celda de flujo. En esas condiciones se mide la "señal de referencia", $\mathrm{S}_{\mathrm{R}}$. Con esas dos medidas se puede definir la escala fotométrica. Para cualquier medida posterior $\mathrm{S}_{\mathrm{X}}$ es posible entonces calcular la absorbancia correspondiente $\mathrm{A}_{\mathrm{X}}$.

Desde el punto de vista analítico, corresponde realizar una calibración analítica para cada serie de medidas. La calibración se realiza midiendo un patrón ubicado en un frasco dentro del gabinete del prototipo. Una vez verificado el rango lineal es posible emplear la calibración por dos puntos, un blanco (realizado con agua destilada) y el patrón mencionado. 
Con los datos de la función de calibración disponibles se puede calcular la concentración correspondiente a cada muestra. Este resultado está entonces disponible para su transmisión a la base mediante el sistema de comunicaciones.

En las etapas de diseño se seleccionó la tecnología de comunicaciones GSM/GPRS por su amplia difusión y cobertura en el país. Las pruebas preliminares realizadas en el laboratorio empleando mensajes de texto resultaron totalmente satisfactorias. En el prototipo final se implementará un sistema más elaborado mediante el uso de internet inalámbrica. Este posibilitará una gestión más avanzada de los datos que podrán ser ingresados directamente a una base de datos.

\section{Consideraciones sobre autonomía}

La autonomía operativa del sistema depende, por un lado, del consumo de reactivos (y la consiguiente generación de residuos) y, por otro, del consumo de electricidad. El subsistema analítico opera mediante la técnica de flujo pulsado, y, por lo tanto, el consumo de reactivos es predecible. En función de esto se ha previsto un ciclo de trabajo de una semana, al final de la cual un operador procederá al recambio de los recipientes de reactivos, patrones y descarte (residuos químicos). Los residuos serán transportados al laboratorio para su adecuada disposición.

De acuerdo a las estimaciones de consumo y al tipo de batería empleada, se prevé que esta última podrá alimentar el sistema sin necesidad de recarga por una semana como mínimo. Sin embargo, esta previsión se basa en una serie de suposiciones que deberán confirmarse en las pruebas de campo. Entre otros aspectos, el comportamiento de las baterías depende de su calidad y del régimen de consumo, y varía a lo largo de su vida útil. En este analizador existen picos de consumo asociados a la operación de las bombas y del módem GSM/GPRS que pueden afectar el comportamiento de la batería a largo plazo. Por estos motivos, si la evaluación de campo demostrase que la duración de la carga de la batería no alcanza el objetivo prefijado, se incorporará como respaldo un panel solar y su circuito asociado para la recarga de la batería. En etapas posteriores se avanzará en la optimización del sistema a fin de lograr una autonomía aún mayor.

\section{CONCLUSIONES}

El prototipo de analizador in situ construido fue evaluado en el laboratorio desde el punto de vista de su desempeño analítico. Los resultados obtenidos en esta primera evaluación resultaron ampliamente satisfactorios con relación a los objetivos propuestos.

Las pruebas de campo en condiciones reales permitirán evaluar en forma definitiva el comportamiento analítico y adicionalmente los aspectos de comunicaciones y autonomía. 


\section{RECONOCIMIENTOS}

A la Comisión Sectorial de Investigación Científica (CSIC-UdelaR) por el financiamiento del proyecto "Desarrollo de una plataforma analítica flexible y de bajo costo para la telemedición de parámetros de calidad de agua". A la Agencia Nacional de Investigación e Innovación (ANII). Al PEDECIBA Química.

\section{REFERENCIAS}

Abbott, D.C., Emsden, G.E. y Harris, J.R., 1963. A method for determining orthophosphate in water. En: Analyst, 88, pp.814-816.

Colaboradores de Wikipedia, [s.d.]. Arduino [En línea]. [s.l.]: Wikipedia. [Consulta: 05 de octubre de 2018]. Disponible en: https://en.wikipedia.org/wiki/Arduino

Glasgow, H.B., Burkholder, J.A.M., Reed, R.E., Lewitus, A.J. y Kleinman, J.E., 2004. Real-time remote monitoring of water quality: a review of current applications, and advancements in sensor, telemetry, and computing technologies. En: Journal of Experimental Marine Biology and Ecology, 300, pp.409-448.

Grotzinger, J.P., Crisp, J., Vasavada, A.R., Anderson, R.C., Baker, C.J., Barry, R., Blake, D.F., Conrad, P., Edgett, K.S., Ferdowski, B., Gellert, R., Gilbert, J.B., Golombek, M., Gómez-Elvira, J., Hassler, D.M., Jandura, L., Litvak, M., Mahaffy, P., Maki, M., Meyer, M., Malin, M.C., Mitrofanov , I., Simmonds, J.J., Vaniman, D.,Welch, R.V. y Wiens, R.C., 2012. Mars science laboratory mission and science investigation. En: Space Science Reviews, 170, pp.5-56.

Estela, J.M. y Cerdà, V., 2005. Flow analysis techniques for phosphorus: an overview. En: Talanta, 66, pp.307-331.

Gründler, P., 2007. Chemical sensors. Berlin: Springer-Verlag.

Hulanicki, A., Glab, S. y Ingman, F., 1991. Chemical sensors. Definitions and classification. En: Pure and Applied Chemisty, 63, pp.1247-1250.

Kitson, R.E., Mellon, M.G., 1944. Colorimetric determination of phosphorus as molybdivanadophosphoric acid. En: Industrial and Engineering Chemistry, Analytical Edition, 16, pp.379-383.

Miró, M., Estela, J.M. y Cerdà, V., 2003. Application of flowing stream techniques to water analysis. Part I. Ionic species: dissolved inorganic carbon, nutrients and related compounds. En: Talanta, 60, pp.867-886.

Motomizu, S. y Li, Z-H., 2005. Trace and ultratrace analysis methods for the determination of phosphorus by flow-injection techniques. En: Talanta, 66, pp 332-340.

Mukhopadhyay, S.C. y Mason, A., eds., 2013. Smart sensors for real-time water quality monitoring. Berlin: Springer-Verlag.

Nagul, E.A., McKelvie, I.D., Worsfold, P., Kolev, S.D., 2015. The molybdenum blue reaction for the determination of orthophosphate revisited: opening the black box. En: Analytica Chimica Acta, 890, pp.60-82.

Narayanaswamy, R. y Wolfbeis, O.S., 2004. Optical sensors. Industrial environmental and diagnostic applications. Berlin: Springer-Verlag. 
Uruguay. Decreto 253/979, de 09 de mayo de 1979. Diario Oficial, 31 de mayo de 1979, No. 20.504, p. 1473.

\section{Página web sugerida}

Arduino: https://www.arduino.cc/ 